\title{
Article
}

\section{Incidence of Intrapartum-Related Events at the Largest Obstetric Hospital in Hanoi, Vietnam: A Retrospective Study}

\author{
Tina Dempsey $1,2, * \mathbb{C}$, Huong Lien Nguyen ${ }^{3}$, Huong Thu Nguyen ${ }^{3}{ }^{\circ}$, Xuan Anh Bui ${ }^{4}$, Phuong Thi Thu Pham ${ }^{3}$, \\ Toan K. Nguyen ${ }^{5,6} \oplus^{\circ}$, Francesco Cavallin ${ }^{7}{ }^{-}$, Daniele Trevisanuto ${ }^{8} \mathbb{D}^{-}$, Susanna Myrnerts Höök ${ }^{1,9}$, \\ Nicolas Pejovic 1,10®, Mats Blennow ${ }^{11}$, Linus Olson ${ }^{1,12,13} \mathbb{D}^{-}$, Hien Vu ${ }^{6,14,15,16}$, Anh Duy Nguyen ${ }^{16,17}$ \\ and Tobias Alfvén ${ }^{1,9}$
}

Citation: Dempsey, T.; Nguyen, H.L.; Nguyen, H.T.; Bui, X.A.; Pham, P.T.T.; Nguyen, T.K.; Cavallin, F.;

Trevisanuto, D.; Myrnerts Höök, S.; Pejovic, N.; et al. Incidence of Intrapartum-Related Events at the Largest Obstetric Hospital in Hanoi, Vietnam: A Retrospective Study. Children 2022, 9, 321. https:// doi.org/10.3390/children9030321

Academic Editor: Bernhard Schwaberger

Received: 12 January 2022

Accepted: 17 February 2022

Published: 28 February 2022

Publisher's Note: MDPI stays neutral with regard to jurisdictional claims in published maps and institutional affiliations.

Copyright: (c) 2022 by the authors. Licensee MDPI, Basel, Switzerland. This article is an open access article distributed under the terms and conditions of the Creative Commons Attribution (CC BY) license (https:// creativecommons.org/licenses/by/ $4.0 /)$.
1 Department of Global Public Health, Karolinska Institutet, 17177 Solna, Sweden; susanna.myrnerts.hook@ki.se (S.M.H.); nicolas.pejovic@ki.se (N.P.); linus.olson@ki.se (L.O.); tobias.alfven@ki.se (T.A.)

2 Astrid Lindgren Children's Hospital, Karolinska University Hospital, 17176 Solna, Sweden

3 Neonatal Department, Phu San Hanoi Hospital, Hanoi 100000, Vietnam; lienhuongnguyenhmu@gmail.com (H.L.N.); nguyenthuhuongkh90@gmail.com (H.T.N.); bsphuong.1971@gmail.com (P.T.T.P.)

4 Department of Information Technology, Phu San Hanoi Hospital, Hanoi 100000, Vietnam; xuanbui88@gmail.com

5 Department of Gynecological Oncology, Phu San Hanoi Hospital, Hanoi 100000, Vietnam; dr.toannguyen@hogh.vn

6 Department of International Collaboration, Phu San Hanoi Hospital, Hanoi 100000, Vietnam; vuhienmd1511@gmail.com

7 Independent Researcher, 36020 Solagna, Italy; cescocava@libero.it

8 Department of Woman's and Child's Health, University Hospital of Padova, 35128 Padova, Italy; daniele.trevisanuto@unipd.it

9 Emergency Care Unit, Sachs' Children and Youth Hospital, 11883 Stockholm, Sweden

10 Neonatal Unit, Sachs' Children and Youth Hospital, 11883 Stockholm, Sweden

11 Department of Clinical Science Intervention and Technology, Karolinska Institutet, 14152 Huddinge, Sweden; mats.blennow@ki.se

12 Department of Women's and Children's Health, Karolinska Institutet, 17177 Solna, Sweden

13 Department of Medical Biochemistry and Microbiology, Uppsala University, 75237 Uppsala, Sweden

14 Intensive Care Unit and Poison Control Department, Phu San Hanoi Hospital, Hanoi 100000, Vietnam

15 Social Work Department, Phu San Hanoi Hospital, Hanoi 100000, Vietnam

16 University of Medicine and Pharmacy, Hanoi 100000, Vietnam; dr.duyanhnguyen@hogh.vn

17 Board of Directors, Phu San Hanoi Hospital, Hanoi 100000, Vietnam

* Correspondence: tina.dempsey@ki.se; Tel.: +46-73-5079-669

\begin{abstract}
Every year, 2.4 million neonates die during their first month of life and even more suffer permanent injury. The main causes are intrapartum-related events, prematurity, and infection, with sub-Saharan Africa and southern Asia being the worst affected regions. With a focus on intrapartumrelated events, we aimed to assess the neonatal demographic characteristics, clinical management, and outcomes among neonates born at the largest obstetric hospital in Hanoi, Vietnam. This was a retrospective cross-sectional study that included all the inborn neonates in November 2019, which was selected as a representative month. A total of 4554 neonates were born during the study period. Of these, $1.0 \%(n=44)$ were stillbirths, $0.15 \%(n=7)$ died in hospital, $0.61 \%(n=28)$ received positive pressure ventilation at birth, and $0.15 \%(n=7)$ were diagnosed with hypoxic ischemic encephalopathy. A total of 581 (13\%) neonates were admitted to the neonatal unit, among which the most common diagnoses were prematurity $(37 \%, n=217)$ and infection $(15 \%, n=89)$. Except for the intrapartum-related events, our findings are consistent with the previously documented data on neonatal morbidity. The intrapartum-related events, however, were surprisingly low in number even in comparison to high-income countries. Research on the current clinical practice at Phu San Hanoi Hospital may bring further clarity to identify the success factors.
\end{abstract}


Keywords: neonatal care; incidence; intrapartum-related events; birth asphyxia; neonatal morbidity; neonatal resuscitation; Vietnam

\section{Background}

Neonatal mortality accounts for nearly half of the 5.3 million deaths that occur every year among children under five years of age [1]. The United Nations (UN) and the World Health Organization (WHO) have, in the last few years, aligned to specifically target neonatal health and end preventable deaths during the newborn period. Their aim is to reduce the overall neonatal mortality to fewer than 12 deaths per 1000 live births in all the countries of the world by 2030 [2].

Complications from prematurity, intrapartum-related events, infection, and congenital anomalies account for approximately $80 \%$ of neonatal mortality globally [1,3]. Intrapartumrelated events originate from the lack of oxygen and subsequent ischemia caused by the disruption of placental blood flow, infection, or when a neonate fails to establish and maintain regular breathing at birth. The immediate damage to the brain results in hypoxic ischemic encephalopathy (HIE) and the risk of neurological sequelae or death [4]. HIE is defined as a clinical syndrome in term neonates. Hypoxic ischemic injury in preterm neonates is complex and lacks a homogenous definition [5]. HIE is diagnosed by the Thompson or Sarnat scoring systems, neuroradiology, and/or amplitude-integrated EEGs. International and local guidelines recommend initiation of hypothermic treatment within six hours from birth for neonates who meet the TOBY cooling criteria [6,7]. Intrapartum-related events are more likely to occur when the antenatal period has been complicated and in multiple or preterm births. Good quality intrapartum care has been recognized as the single most important factor in preventing intrapartum complications $[8,9]$. Once a neonate with respiratory depression is born, prompt drying, stimulation, and positive pressure ventilation (PPV) are the most important interventions [10]. On a global level, intrapartum-related events account for approximately 700,000 neonatal deaths every year [3]. However, neonatal mortality estimates only include live births, and it has been reported that approximately $50 \%$ of all stillbirths are due to an intrapartum-related event [11].

Prematurity-related death is mainly mediated via respiratory distress, feeding issues, and infection. The outcome of the affected neonate is greatly improved with the use of antenatal corticosteroids, kangaroo mother care, surfactant, continuous positive airway pressure (CPAP), feeding tubes, and total parenteral nutrition [12-14]. Infections can be congenital or acquired $[15,16]$. Congenital infections can be transmitted from mother to neonate during pregnancy (transplacental) or during birth (intrapartum). Neonatal sepsis can be defined as early or late onset, with early onset sepsis being more likely congenital. Early onset within the setting of a neonatal unit is defined as $<72 \mathrm{~h}$ of life [17]. The key aspects of successfully reducing neonatal mortality secondary to infection are the identification and minimization of risk factors, supportive treatment, and adequate antibiotic/antiviral therapy. Congenital anomalies are estimated to be the fourth most common cause of neonatal death, and they contribute to a significant proportion of neonatal disease [18]. Their distribution is variable depending on both genetic and environmental factors. The rate of antenatal diagnostics and abortion highly influence the rate of congenital anomalies [19,20].

The reported rates of neonatal mortality in Vietnam are relatively good compared to other low- and middle-income countries (LMIC). The World Bank reported 10.5 deaths/1000 live births in 2019 [21]. However, a study from 2010 highlighted a substantial under-reporting of neonatal mortality in the official statistics, showing the neonatal mortality rate $(16 / 1000)$ in Quang Ninh province to be fourfold higher than the rate reported to the Ministry of Health $(4.2 / 1000)$ [22]. The WHO Every Newborn Action Plan (ENAP) highlights the importance of improving data collection to identify gaps in equity and quality. Its progress report in 2018 identified Vietnam as being one of a number of countries that had no perinatal death review system in place [23]. With a focus on intrapartum-related events, we aimed to assess 
the neonatal demographics, clinical management, and outcomes among neonates born at the largest obstetric hospital in Hanoi, the capital of Vietnam. The results of this study should raise awareness regarding the main causes of neonatal mortality and morbidity in urban Vietnam and how they compare to the primary causes on a global scale.

\section{Methods}

\subsection{Study Setting}

With approximately 40,000 births annually, Phu San Hanoi Hospital is the largest obstetric hospital in Hanoi and the top government public hospital in Vietnam. It is situated next to Vietnam National Children's Hospital (VNCH). The hospital's overall c-section rate is greater than $50 \%$, approximately half of which are emergency c-sections. Most newborn neonates are admitted to the postnatal ward for $24 \mathrm{~h}$ before being discharged. Neonates who are born with clinical distress and/or risk factors are admitted to the neonatal unit, which is staffed by 72 nurses and 21 doctors. The neonatal unit at Phu San Hanoi Hospital is divided into a neonatal intensive care unit (NICU; 38 beds and 29 incubators) and a high dependency unit (50 beds).

Many structural and clinical changes have taken place at Phu San Hanoi Hospital during the past few years. Birth attendants who assisted 4-6 birthing women simultaneously 5 years ago now assist 1-2 simultaneous vaginal deliveries. The following therapies are currently available at Phu San Hospital: mechanical ventilation (available since 1996), surfactant therapy (2000), CPAP (2001), high frequency oscillatory ventilation (2012), umbilical venous catheterization (2012), umbilical arterial catheterization (2015), and minimally invasive surfactant administration techniques (Insure 2016, Lisa 2018). Admission criteria to the neonatal unit include: preterm birth $<34$ weeks, low birth weight $<2000$ g, neonates with $\geq 2$ risk factors (maternal fever, stained or foul-smelling amniotic fluid, prolonged rupture of membranes $\geq 18 \mathrm{~h}$, premature rupture of membranes, maternal colonization by group B streptococcus), maternal insulin dependent diabetes mellitus, need for PPV at birth, respiratory failure, and congenital anomalies.

Neonates who require surgery (including severe pneumothorax), blood exchange transfusion, cooling or ECMO, or suffer from severe pulmonary hypertension, multiresistant bacteremia, congenital metabolic disorders, or, rarely, on family request, are transferred to the neighboring hospital's neonatal unit at $\mathrm{VNCH}$.

\subsection{Study Design}

This was a retrospective cross-sectional study including all the inborn neonates at Phu San Hanoi Hospital in November 2019. The number of livebirths, stillbirths, and neonatal unit admissions was identified using the local electronic system. Liveborn neonates deceased prior to admission to the neonatal unit were identified from the local mortality book. Data related to the neonates admitted to the neonatal unit were extracted from the neonates' medical files by two neonatologists using a standardized hard copy case report form (CRF). The variables of interest included: maternal and study subject demographics, perinatal risk factors, medical diagnoses, clinical management, and outcome. Data were transferred from the CRFs into an electronic database (RedCap, Vanderbilt, TN, USA) by two non-clinical data officers. Before data transfer, the officers completed a one-day orientation and training that focused on data entry into RedCap from clinical CRFs. For quality control purposes, $5 \%$ of the transferred data were double checked by the project coordinator.

The definitions used include: prematurity $<37$ weeks gestation, hypoglycemia $<2.6 \mathrm{mmol} / \mathrm{L}$, hypothermia $<36{ }^{\circ} \mathrm{C}$, and hyperbilirubinemia $>120 \mathrm{mmol} / \mathrm{L}(>7 \mathrm{mg} / \mathrm{dL})$. Bronchopulmonary dysplasia was defined as oxygen dependency at 36 weeks' gestation (for neonates $<32$ weeks), or as oxygen dependency at 29-55 days of life (for neonates $\geq 32$ weeks). Respiratory distress syndrome (RDS) was diagnosed in preterm neonates who required supplemental oxygen, CPAP, or mechanical ventilation, and when the respiratory distress could not be explained by another diagnosis. Surfactant was administered based on the European Consensus Guidelines on the Management of RDS-2019 [24]. HIE was diagnosed 
in term as well as preterm neonates based on clinical exam by the Sarnat scoring system in neonates that fulfilled one of the asphyxia criteria: Apgar score $<5$ at 10 min of life, $\mathrm{pH}<7.0$ within the first hour of life, or base excess $<-16 \mathrm{mmol} / \mathrm{L}$ within the first hour of life. Both confirmed (culture positive) and suspected infections (based on risk factors, clinical exam, blood tests, and radiology) were recorded. Infection was defined as congenital if an identified pathogen was known to be vertically transmitted (e.g., TORCH infection) or if the infection was diagnosed within the first $72 \mathrm{~h}$ of life. We defined metabolic disease and tumors as congenital anomalies according to the WHO's definition (a structural or functional anomaly that occurs during intrauterine life) [18]. The indication for antibiotic administration was determined based on a hierarchal level where the highest valid cause was documented (culture confirmed > elevated infectious markers and/or suspicious chest $X$-ray $>$ known risk factors/suspicious clinical exam $>$ unknown).

\subsection{Ethical Approval}

The study was approved by the Vietnamese Institutional Review Board in Human Research Dinh Tien Hoang Institute of Medicine (IRB-2001) on 26 March 2020. The data analysis took place in Sweden and was approved by the Swedish Ethical Review Authority (Dnr 2021-00064) on 5 March 2021.

\subsection{Statistical Analysis}

Variables were reported as frequencies and percentages for categorical data, and medians with interquartile ranges (IQR) for continuous data. The variables were compared among birthweight categories using a chi-square test and Fisher's exact test (categorical variables), and Kruskal-Wallis (continuous variables). A p-value less than 0.05 was considered significant. Data analysis was performed using STATA version 16.1 (StataCorp LCC, College Station, TX, USA).

\section{Results}

A total of 4554 neonates were born at the hospital in November 2019. Of these, $1.0 \%$ were stillbirths $(n=44)$ and $13 \%(n=581)$ were admitted to the neonatal unit. There were no deaths of liveborn neonates prior to neonatal unit admission (Figure 1).

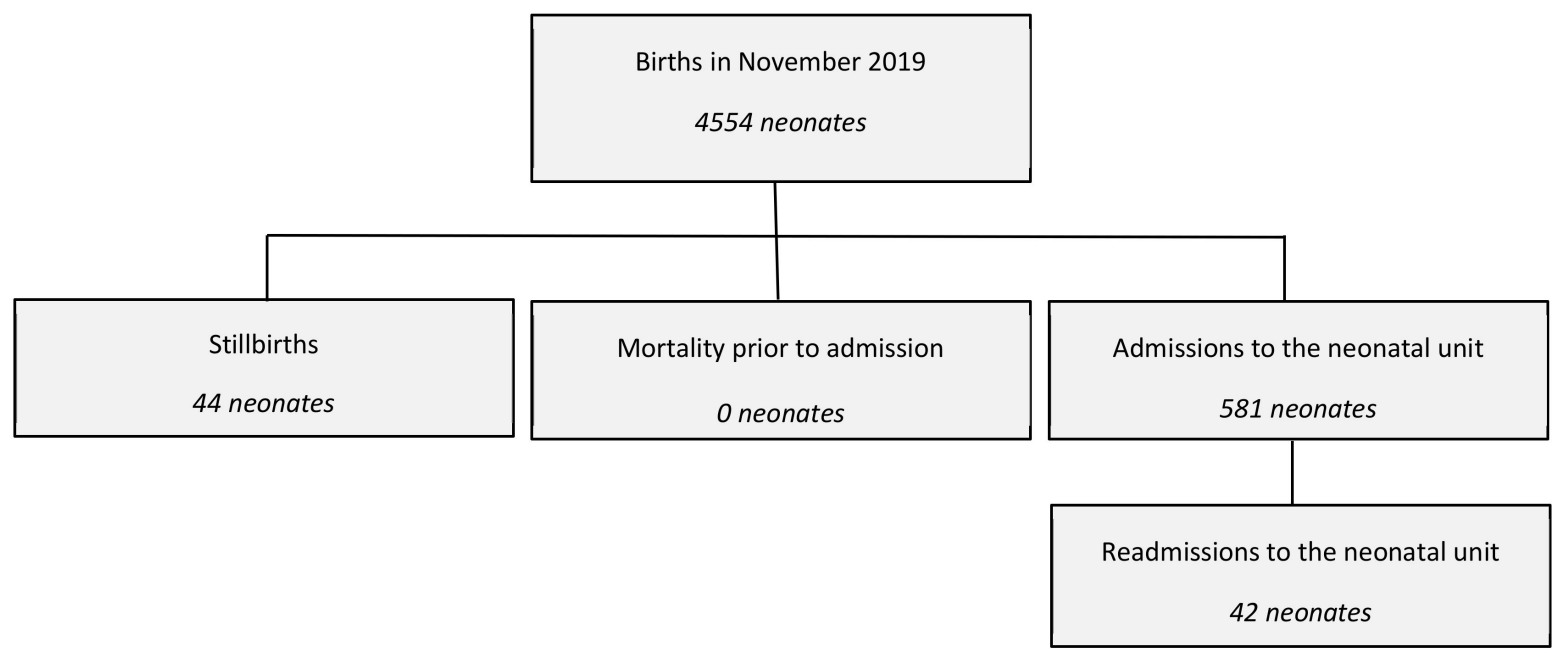

Figure 1. Flow chart of neonates included in the study.

3.1. Demography, Mode of Delivery, and Resuscitation of Neonates Admitted to the Neonatal Unit

The full demographic details are presented in Table 1. Of the neonates admitted to the neonatal unit, 37\% were preterm, $2.9 \%$ were extremely preterm ( $<28$ weeks' gestation), and $1.9 \%$ had an extremely low birth weight (ELBW, $<1000 \mathrm{~g})$. Approximately $60 \%$ were delivered by c-section, and only one neonate had an instrumental delivery (forceps). At 
birth, the overall PPV rate was $0.6 \%$ ( $n=28 / 4554$ births), and, among the neonates admitted to the neonatal unit, $4.8 \%$. The overall incidence of endotracheal intubations (ETI) was $0.22 \%(10 / 4554$ births), and the proportion of intubation among the neonates receiving PPV was $36 \%$ (10/28 neonates). The neonates with a lower birth weight had a significantly higher incidence of 5-min Apgar $<7$. This group also required significantly more PPV, including PPV delivered via ETI.

Table 1. Demography, mode of delivery, and resuscitation of neonates admitted to the neonatal unit at Phu San Hospital in November 2019.

\begin{tabular}{|c|c|c|c|c|c|c|c|c|}
\hline & \multicolumn{2}{|c|}{$\begin{array}{l}\text { All Neonates } \\
\qquad N=581\end{array}$} & \multicolumn{2}{|c|}{$\begin{array}{c}\text { Birth Weight }^{\text {a }} \\
\quad<1500 \mathrm{~g} \\
N=57\end{array}$} & \multicolumn{2}{|c|}{$\begin{array}{c}\text { Birth Weight }{ }^{\text {a }} \\
1500-2500 \mathrm{~g} \\
N=167\end{array}$} & \multicolumn{2}{|c|}{$\begin{array}{c}\text { Birth Weight }{ }^{\text {a }} \\
\quad>2500 \mathrm{~g} \\
N=353\end{array}$} \\
\hline & $n /$ Median & $\% / I Q R$ & $n /$ Median & $\% / I Q R$ & $n /$ Median & $\% / I Q R$ & $n /$ Median & $\% / I Q R$ \\
\hline \multicolumn{9}{|l|}{ General } \\
\hline Female & 256 & 44.1 & 24 & 42.1 & 83 & 49.7 & 148 & 41.9 \\
\hline Multiple births & 101 & 17.4 & 23 & 40.4 & 55 & 32.9 & 22 & 6.2 \\
\hline Maternal factors & & & & & & 0.0 & & \\
\hline Age (years) & 29 & $26-33$ & 28 & $26-31$ & 29 & $26-33$ & 29 & $26-33$ \\
\hline Primipara & 270 & 46.5 & 35 & 61.4 & 83 & 49.7 & 151 & 42.8 \\
\hline Previous preterm births & 14 & 2.4 & 0 & 0.0 & 3 & 1.8 & 11 & 3.1 \\
\hline $\begin{array}{c}\text { Previous miscarriage/stillbirth } \\
\text { Perinatal factors }\end{array}$ & 78 & 13.4 & 6 & 10.5 & 21 & 12.6 & 51 & 14.4 \\
\hline Stained/foul-smelling meconium & 22 & 3.8 & 1 & 1.8 & 7 & 4.2 & 14 & 4.0 \\
\hline PROM $^{\mathrm{b}}$ & 95 & 16.4 & 14 & 24.6 & 41 & 24.6 & 41 & 11.6 \\
\hline Maternal infection during labour & 6 & 1.0 & 2 & 3.5 & 2 & 1.2 & 2 & 0.6 \\
\hline Maternal temp. $<36^{\circ} \mathrm{C}$ or $>38^{\circ} \mathrm{C}$ & 2 & 0.3 & 0 & 0.0 & 0 & 0.0 & 2 & 0.6 \\
\hline Preeclampsia & 26 & 4.5 & 4 & 7.0 & 16 & 9.6 & 6 & 1.7 \\
\hline Eclampsia & 1 & 0.0 & 0 & 0.0 & 0 & 0.0 & 1 & 0.0 \\
\hline Placenta praevia & 16 & 2.8 & 3 & 5.3 & 3 & 1.8 & 10 & 2.8 \\
\hline Placenta abruption & 4 & 0.7 & 1 & 1.8 & 0 & 0.0 & 3 & 0.8 \\
\hline \multicolumn{9}{|l|}{ Mode of delivery } \\
\hline Spontaneous vaginal delivery & 233 & 40.1 & 37 & 64.9 & 62 & 37.1 & 133 & 37.7 \\
\hline Assisted (forceps or vacuum) & 1 & 0.2 & 0 & 0.0 & 0 & 0.0 & 1 & 0.3 \\
\hline Elective c-section & 225 & 38.7 & 9 & 15.8 & 57 & 34.1 & 159 & 45.0 \\
\hline Emergency c-section & 119 & 20.5 & 11 & 19.3 & 48 & 28.7 & 60 & 17.0 \\
\hline \multicolumn{9}{|l|}{ Gestational age (weeks') c } \\
\hline$<28$ & 17 & 2.9 & 17 & 29.8 & 0 & 0.0 & 0 & 0.0 \\
\hline $28-32$ & 32 & 5.5 & 23 & 40.4 & 9 & 5.4 & 0 & 0.0 \\
\hline $32-37$ & 168 & 28.9 & 16 & 28.1 & 111 & 66.5 & 41 & 11.6 \\
\hline$>37$ & 359 & 61.8 & 1 & 1.8 & 46 & 27.5 & 311 & 88.1 \\
\hline \multicolumn{9}{|l|}{ Resuscitation } \\
\hline Apgar score at $1 \mathrm{~min}$ & 8 & $7-8$ & 6 & $5-6.5$ & 7 & $7-8$ & 8 & $8-8$ \\
\hline Apgar score at $5 \mathrm{~min}$ & 9 & $8-9$ & 7 & $6-7.5$ & 8 & $8-9$ & 9 & 9-9 \\
\hline Apgar score $<7$ at $5 \mathrm{~min}$ & 30 & 5.2 & 22 & 38.6 & 3 & 1.8 & 4 & 1.1 \\
\hline Face mask ventilation ${ }^{\mathrm{d}}$ & 26 & 4.5 & 15 & 26.3 & 9 & 5.4 & 2 & 0.6 \\
\hline Endotracheal intubation & 10 & 1.7 & 3 & 5.3 & 4 & 2.4 & 3 & 0.8 \\
\hline
\end{tabular}

${ }^{a}$ Four neonates missing. ${ }^{b}$ Combines preterm and term premature rupture of membrane (PROM). ${ }^{c}$ Five neonates missing. ${ }^{\mathrm{d}}$ Includes eight neonates who also received endotracheal intubation.

\subsection{Diagnoses in Neonates Admitted to Neonatal Unit}

Table 2 reports the diagnoses for the neonates admitted to the neonatal unit, both overall and in relation to birth weight. Consistent with the need for PPV, HIE was more common in the very low birth weight (VLBW) neonates $(7.0 \%, n=4)$ compared to low birth weight (LBW) neonates $(1.2 \%, n=2)$ and normal birth weight (NBW) neonates $(0.3 \%, n=1)$. Only one neonate was diagnosed with seizures, which appears under "other" diagnoses. 
Table 2. Diagnoses in all neonates admitted to the neonatal unit (first admission) at Phu San Hospital in November 2019.

\begin{tabular}{|c|c|c|c|c|c|c|c|c|}
\hline & \multicolumn{2}{|c|}{$\begin{array}{l}\text { All Neonates } \\
\qquad N=581\end{array}$} & \multicolumn{2}{|c|}{$\begin{array}{c}\text { Birth Weight }^{\mathrm{a}} \\
\quad<\mathbf{1 5 0 0 \mathrm { g }} \\
\quad N=57\end{array}$} & \multicolumn{2}{|c|}{$\begin{array}{c}\text { Birth Weight }{ }^{\text {a }} \\
1500-2500 \mathrm{~g} \\
N=167\end{array}$} & \multicolumn{2}{|c|}{$\begin{array}{c}\text { Birth Weight } \\
\quad>2500 \mathrm{~g} \\
N=353\end{array}$} \\
\hline & $n$ & $\%^{b}$ & $n$ & $\%^{b}$ & $n$ & $\%^{b}$ & $n$ & $\%^{b}$ \\
\hline \multicolumn{9}{|l|}{ Birth weight and gestational age } \\
\hline Prematurity & 217 & 37.3 & 56 & 98.2 & 120 & 71.9 & 41 & 11.6 \\
\hline Small for gestational age & 44 & 7.6 & 12 & 21.1 & 30 & 18.0 & 2 & 0.6 \\
\hline Large for gestational age & 4 & 0.7 & 0 & 0.0 & 1 & 0.6 & 3 & 0.8 \\
\hline \multicolumn{9}{|l|}{ Cardiopulmonary } \\
\hline Respiratory distress syndrome & 96 & 16.5 & 47 & 82.5 & 33 & 19.8 & 15 & 4.2 \\
\hline Respiratory distress resolved within $6 \mathrm{~h}$ & 60 & 10.3 & 6 & 10.5 & 33 & 19.8 & 21 & 5.9 \\
\hline Transient tachypnoea of the newborn & 23 & 4.0 & 0 & 0.0 & 6 & 3.6 & 17 & 4.8 \\
\hline Cyanotic attack & 23 & 4.0 & 0 & 0.0 & 2 & 1.2 & 21 & 5.9 \\
\hline Patent ductus arteriosus & 11 & 1.9 & 8 & 14.0 & 3 & 1.8 & 0 & 0.0 \\
\hline Apnea requiring treatment & 8 & 1.4 & 6 & 10.5 & 2 & 1.2 & 0 & 0.0 \\
\hline Bronchopulmonary dysplasia & 4 & 0.7 & 4 & 7.0 & 0 & 0.0 & 0 & 0.0 \\
\hline Arrhythmia ${ }^{c}$ & 4 & 0.7 & 0 & 0.0 & 0 & 0.0 & 4 & 1.1 \\
\hline Persistent pulmonary hypertension & 3 & 0.5 & 0 & 0.0 & 0 & 0.0 & 3 & 0.8 \\
\hline Pleural effusion & 3 & 0.5 & 0 & 0.0 & 2 & 1.2 & 1 & 0.3 \\
\hline \multicolumn{9}{|l|}{ Hospital acquired infection } \\
\hline $\begin{array}{l}\text { All combined } \\
\text { Diagnosis: }\end{array}$ & 47 & 8.1 & 39 & 68.4 & 5 & 3.0 & 3 & 0.8 \\
\hline Suspected based on other parameters & 23 & 4.0 & 16 & 28.1 & 4 & 2.4 & 3 & 0.8 \\
\hline Culture confirmed & 16 & 2.8 & 16 & 28.1 & 0 & 0.0 & 0 & 0.0 \\
\hline $\begin{array}{l}\text { Chest } X \text {-ray confirmed pneumonia } \\
\text { Congenital infection }\end{array}$ & 8 & 1.4 & 7 & 12.3 & 1 & 0.6 & 0 & 0.0 \\
\hline All combined & 42 & 7.2 & 5 & 8.8 & 12 & 7.2 & 25 & 7.1 \\
\hline High risk based on other parameters & 19 & 3.3 & 2 & 3.5 & 3 & 1.8 & 14 & 4.0 \\
\hline TORCH infection & 6 & 1.0 & 1 & 1.8 & 3 & 1.8 & 2 & 0.6 \\
\hline Maternal syphilis or HIV & 5 & 0.9 & 0 & 0.0 & 1 & 0.6 & 4 & 1.1 \\
\hline Prolonged ROM & 5 & 0.9 & 0 & 0.0 & 4 & 2.4 & 1 & 0.3 \\
\hline & \multicolumn{3}{|c|}{ Intrapartum-related event } & 3.5 & 1 & 0.6 & 4 & 1.1 \\
\hline PPV requirement & 28 & 4.8 & 15 & 26.3 & 9 & 5.4 & 4 & 1.1 \\
\hline Hypoxic ischaemic encephalopathy & 7 & 1.2 & 4 & 7.0 & 2 & 1.2 & 1 & 0.3 \\
\hline $\begin{array}{l}\text { Birth related trauma } \mathrm{d} \\
\text { Gastrointestinal }\end{array}$ & 4 & 0.7 & 0 & 0.0 & 1 & 0.6 & 3 & 0.8 \\
\hline Discolored vomiting & 26 & 4.5 & 1 & 1.8 & 0 & 0.0 & 25 & 7.1 \\
\hline Other minor disturbance & 6 & 1.0 & 0 & 0.0 & 0 & 0.0 & 6 & 1.7 \\
\hline $\begin{array}{c}\text { Necrotizing enterocolitis or obstruction } \\
\text { Other }\end{array}$ & 5 & 0.9 & 1 & 1.8 & 1 & 0.6 & 3 & 0.8 \\
\hline Jaundice & 184 & 31.7 & 36 & 63.2 & 64 & 38.3 & 83 & 23.5 \\
\hline Maternal diabetes mellitus & 78 & 13.4 & 0 & 0.0 & 13 & 7.8 & 65 & 18.4 \\
\hline Congenital anomaly $\mathrm{e}$ & 67 & 11.5 & 3 & 5.3 & 13 & 7.8 & 51 & 14.4 \\
\hline Other $\mathrm{f}$ & 30 & 5.2 & 2 & 3.5 & 8 & 4.8 & 20 & 5.7 \\
\hline Intraventricular hemorrhage & 3 & 0.5 & 3 & 5.3 & 0 & 0.0 & 0 & 0.0 \\
\hline
\end{tabular}

${ }^{a}$ Four neonates missing. ${ }^{b}$ Some neonates have more than one diagnosis. Proportions (\%) are presented in reference to the number of neonates. ${ }^{\mathrm{c}}$ Includes: bradycardia $(n=3)$ and extrasystole $(n=1) .{ }^{\mathrm{d}}$ Includes: clavicular fracture $(n=2)$; facial nerve injury following forceps usage $(n=1)$; large cephalohematoma $(n=1) .{ }^{\mathrm{e}}$ Includes: cardiovascular $(n=32)$; gastrointestinal $(n=10)$; pulmonary $(n=9)$; suspected or confirmed syndrome $(n=5)$ dysmelia $(n=2)$; renal $(n=1)$; neurological $(n=1)$; metabolic $(n=1)$; tumour $(n=1)$; unknown $(n=5) .{ }^{\mathrm{f}}$ Includes all the conditions for which $n \leq 2$ or diagnoses that are no longer indication for admission.

\subsection{Clinical Management of Neonates Admitted to the Neonatal Unit}

The most common therapies at the neonatal unit were: use of a feeding tube $(54 \%$, $n=313)$, phototherapy $(31 \%, n=180)$, antibiotic administration $(24 \%, n=140)$, and oxygen supplementation $(23 \%, n=134)$ (Table 3$)$. One neonate was transferred to $\mathrm{VNCH}$ for 
therapeutic hypothermia. All the ELBW neonates who survived their first day of life $(n=10)$ received antibiotics, total parenteral nutrition, and caffeine. Ninety percent of the ELBW neonates received non-invasive respiratory support (CPAP). Less than one-third of all the neonates with RDS received surfactant $(n=31)$. Eight neonates received surfactant without ever requiring mechanical/oscillatory ventilation.

Table 3. Clinical management of all neonates admitted to the neonatal unit (first admission) at Phu San Hospital in November 2019.

\begin{tabular}{|c|c|c|c|c|c|c|c|c|}
\hline & \multicolumn{2}{|c|}{$\begin{array}{l}\text { All Neonates } \\
\qquad N=581\end{array}$} & \multicolumn{2}{|c|}{$\begin{array}{c}\text { Birth Weight }^{\mathrm{a}} \\
\quad<1500 \mathrm{~g} \\
N=57\end{array}$} & \multicolumn{2}{|c|}{$\begin{array}{c}\text { Birth Weight }^{\text {a }} \\
1500-2500 \mathrm{~g} \\
N=167\end{array}$} & \multicolumn{2}{|c|}{$\begin{array}{c}\text { Birth Weight }^{\text {a }} \\
\quad>2500 \mathrm{~g} \\
N=353\end{array}$} \\
\hline & $n /$ Median & $\% / I Q R$ & $n /$ Median & $\% / I Q R$ & $n /$ Median & $\% / I Q R$ & $n /$ Median & $\% / I Q R$ \\
\hline \multicolumn{9}{|l|}{ Antibiotics } \\
\hline $\begin{array}{l}\text { All causes combined } \\
\text { Indication: }\end{array}$ & 140 & 24.1 & 50 & 87.7 & 43 & 25.7 & 46 & 13.0 \\
\hline Based on clinical risk factors & 91 & 15.7 & 22 & 38.6 & 37 & 22.2 & 31 & 8.8 \\
\hline Based on blood tests ${ }^{b}$ and radiology & 31 & 5.3 & 11 & 19.3 & 6 & 3.6 & 14 & 4.0 \\
\hline Culture-confirmed & 17 & 2.9 & 16 & 28.1 & 0 & 0.0 & 1 & 0.3 \\
\hline Unknown & 1 & 0.2 & 1 & 1.8 & 0 & 0.0 & 0 & 0.0 \\
\hline \multicolumn{9}{|l|}{ Respiratory } \\
\hline Oxygen therapy & 134 & 23.1 & 41 & 71.9 & 55 & 32.9 & 37 & 10.5 \\
\hline CPAP & 58 & 10.0 & 33 & 57.9 & 18 & 10.8 & 6 & 1.7 \\
\hline Mechanical ventilation & 46 & 7.9 & 18 & 31.6 & 10 & 6.0 & 17 & 4.8 \\
\hline Oscillatory ventilation ${ }^{c}$ & 9 & 1.5 & 6 & 10.5 & 1 & 0.6 & 2 & 0.6 \\
\hline Duration of ventilation (days) & 4 & $2-9$ & 9 & $7-14$ & 4 & $2-4$ & 2 & $1-2$ \\
\hline Caffeine administration & 68 & 11.7 & 44 & 77.2 & 22 & 13.2 & 0 & 0.0 \\
\hline Surfactant administration & 31 & 5.3 & 17 & 29.8 & 11 & 6.6 & 2 & 0.6 \\
\hline \multicolumn{9}{|l|}{ Fluids, electrolytes, and nutrition } \\
\hline Feeding tube & 313 & 53.9 & 48 & 84.2 & 52 & 31.1 & 30 & 8.5 \\
\hline Glucose infusion & 82 & 14.1 & 8 & 14.0 & 34 & 20.4 & 40 & 11.3 \\
\hline Total parenteral nutrition & 58 & 10.0 & 44 & 77.2 & 11 & 6.6 & 2 & 0.6 \\
\hline \multicolumn{8}{|l|}{ General } & 19.5 \\
\hline Analgesia administration & 16 & 2.8 & 3 & 5.3 & 6 & 3.6 & 7 & 2.0 \\
\hline Phototherapy & 180 & 31.0 & 40 & 70.2 & 59 & 35.3 & 81 & 22.9 \\
\hline Inotropic drugs administration & 12 & 2.1 & 7 & 12.3 & 1 & 0.6 & 4 & 1.1 \\
\hline Gastric lavage & 3 & 0.5 & 0 & 0.0 & 0 & 0.0 & 3 & 0.8 \\
\hline Otrivin administration & 1 & 0.2 & 0 & 0.0 & 0 & 0.0 & 1 & 0.3 \\
\hline
\end{tabular}

${ }^{a}$ Four neonates missing. ${ }^{b}$ Excludes blood culture-confirmed infection. ${ }^{c}$ All nine neonates also received mechanical ventilation.

\subsection{Outcome of Neonates Admitted to the Neonatal Unit}

The outcomes are presented in Table 4 . The overall mortality rate at Phu San neonatal unit (excluding neonates discharged to VNCH or other neonatal units) was $0.2 \%(n=7)$, of whom five had a birth weight $<1000 \mathrm{~g}$, one died from a congenital diaphragmatic hernia, and one died from complications due to metabolic disease. Just over half $(53 \%, n=310)$ of the neonates admitted to the neonatal unit were discharged within $24 \mathrm{~h}$ of admission, with $6.7 \%(n=39)$ being transferred to $\mathrm{VNCH}$ or another hospital. A total of $13 \%(n=73)$ of the admitted neonates were transferred to $\mathrm{VNCH}$ or another hospital. Amongst the neonates not discharged within their first $24 \mathrm{~h}$, the median duration of admission was 6 (IQR 3-13) days, with the lower birth weight neonates having a significantly longer stay. A total of $6.9 \%(n=40)$ were readmitted after discharge, with phototherapy $(90 \%, n=36)$ being the most common indication for readmission. 
Table 4. Clinical outcomes among all neonates admitted to the neonatal unit at Phu San Hospital in November 2019.

\begin{tabular}{|c|c|c|c|c|c|c|c|c|}
\hline & \multicolumn{2}{|c|}{$\begin{array}{l}\text { All Neonates } \\
\qquad N=581\end{array}$} & \multicolumn{2}{|c|}{$\begin{array}{c}\text { Birth Weight }^{\mathrm{a}} \\
\quad<1500 \mathrm{~g} \\
\quad N=57\end{array}$} & \multicolumn{2}{|c|}{$\begin{array}{c}\text { Birth Weight }{ }^{a} \\
1500-2500 \mathrm{~g} \\
N=167\end{array}$} & \multicolumn{2}{|c|}{$\begin{array}{l}\text { Birth Weight }^{\mathrm{a}} \\
\quad>2500 \mathrm{~g} \\
N=353\end{array}$} \\
\hline & $n /$ Median & $\% / I Q R$ & $n /$ Median & $\% / I Q R$ & $n /$ Median & $\% / I Q R$ & $n /$ Median & $\% / I Q R$ \\
\hline \multicolumn{9}{|l|}{ First admission } \\
\hline Number of neonates & 581 & 100.0 & 57 & 100.0 & 167 & 100.0 & 353 & 100.0 \\
\hline Duration $<1$ day & 310 & 53.4 & 4 & 7.0 & 76 & 45.8 & 230 & 65.0 \\
\hline \multicolumn{8}{|l|}{ Readmission } & $2-5$ \\
\hline Number of neonates & 40 & 6.9 & 1 & 1.8 & 19 & 11.4 & 20 & 5.6 \\
\hline Number of readmissions & $42^{c}$ & - & 1 & - & 21 & - & 20 & - \\
\hline Duration $<1$ day & 7 & 17.5 & 0 & 0.0 & 4 & 15.8 & 4 & 20.0 \\
\hline If $>1$ day, total duration (days) & 4 & $3-4$ & 3 & $3-3$ & 3 & $3-4$ & 3 & $2-4$ \\
\hline Phototherapy & 36 & 90.0 & 1 & 100.0 & 18 & 94.7 & 17 & 85.0 \\
\hline Antibiotic therapy & 3 & 7.5 & 0 & 0.0 & 2 & 10.5 & 1 & 5.0 \\
\hline Feeding tube & 2 & 5.0 & 0 & 0.0 & 2 & 10.5 & 0 & 0.0 \\
\hline Glucose infusion & 1 & 2.5 & 0 & 0.0 & 0 & 0.0 & 1 & 5.0 \\
\hline Oxygen therapy & 1 & 2.5 & 0 & 0.0 & 1 & 5.3 & 0 & 0.0 \\
\hline \multicolumn{9}{|l|}{ Quality of care parameters } \\
\hline Number of admissions & 623 & 100.0 & 58 & 100.0 & 187 & 100.0 & 374 & 100.0 \\
\hline Documentation of vital parameters ${ }^{d}$ & 603 & 96.8 & 56 & 96.6 & 186 & 99.5 & 361 & 96.5 \\
\hline Initial hypothermia & 14 & 2.3 & 9 & 16.1 & 1 & 0.5 & 4 & 1.1 \\
\hline Improved to $>36{ }^{\circ} \mathrm{C}$ within $2 \mathrm{~h}$ & 8 & 57.1 & 7 & 77.8 & 0 & 0.0 & 1 & 25.0 \\
\hline Documentation of blood glucose ${ }^{\mathrm{d}}$ & 358 & 57.5 & 47 & 81.0 & 133 & 71.1 & 178 & 47.6 \\
\hline Initial hypoglycemia & 41 & 11.5 & 7 & 14.9 & 18 & 13.5 & 16 & 9.0 \\
\hline $\begin{array}{c}\text { Improved to }>2.6 \mathrm{mmol} / \mathrm{L} \\
\text { within } 30 \mathrm{~min}\end{array}$ & 37 & 90.2 & 7 & 100.0 & 15 & 83.3 & 15 & 93.8 \\
\hline \multicolumn{9}{|l|}{ Final discharge location e } \\
\hline Home & 221 & 38.0 & 30 & 52.6 & 80 & 48.2 & 110 & 31.1 \\
\hline Postnatal ward Phu San & 267 & 46.0 & 3 & 5.3 & 69 & 41.6 & 195 & 55.1 \\
\hline $\mathrm{VNCH}$ & 49 & 8.4 & 11 & 19.3 & 12 & 7.2 & 26 & 7.3 \\
\hline Other hospital & 24 & 4.1 & 4 & 7.0 & 3 & 1.8 & 17 & 4.8 \\
\hline If discharged to & & & & & & & & \\
\hline $\begin{array}{l}\text { VNCH/other hospital, } \\
\text { discharged within } 24 \mathrm{~h}^{\mathrm{f}} \\
\text { Mortality }\end{array}$ & 39 & 53.4 & 0 & 0.0 & 4 & 26.7 & 35 & 81.4 \\
\hline Deaths & 7 & 1.2 & 5 & 8.8 & 0 & 0.0 & 2 & 0.6 \\
\hline Timing (days of life) & 7 & $1-20$ & 10 & $7-20$ & - & - & 3 & $1-5$ \\
\hline
\end{tabular}

${ }^{a}$ Four neonates missing. ${ }^{b}$ Seven neonates missing. ${ }^{c}$ One neonate was readmitted three times. ${ }^{\mathrm{d}}$ Documentation within 30 min of admission (vital parameters $=$ heart rate, $\mathrm{SpO}_{2}$, and body temperature). ${ }^{\mathrm{e}}$ Twenty neonates missing. ${ }^{\mathrm{f}}$ Three neonates missing.

\subsection{Quality Control}

We found a $0.6 \%$ mismatch between the hard copy CRFs and the RedCap insertions.

\section{Discussion}

This study showed a lower-than-expected rate of intrapartum-related events at Phu San Hanoi Hospital, whereas the main causes of neonatal morbidity and mortality otherwise mirrored those causes on a global scale. The overall PPV rate was $0.61 \%$, and approximately one-third of those patients progressed to ETIs. A global review from 2011 estimated that 3 to $6 \%$ of newborn neonates require PPV at birth, and $<1 \%$ require ETI [25]. A study from the United States including neonates with a gestational age $\geq 34$ weeks found that $6 \%$ required PPV at birth, and, of these, $22 \%$ received ETI [26]. Two Norwegian studies reported an overall PPV rate of $3 \%$ and $4 \%$, respectively, with ETI requirements of $9 \%$ and $6.9 \%$. The latter showed a higher PPV rate in neonates $<34$ weeks $(21 \%)$ compared to $>34$ weeks $(3 \%)[27,28]$. 
A Brazilian study that looked at neonates with NBW without a congenital anomaly, reported a PPV rate of 3.1\% [29]. Research conducted at a hospital in Tanzania showed that $6.5 \%$ of the newborn neonates needed PPV at birth [30]. Finally, research conducted in Uganda by part of our own research team found that $8.6 \%$ of the included neonates (gestational age $\geq 34$ weeks and birth weight $\geq 2000 \mathrm{~g}$ ) required PPV at birth [31].

The incidence of HIE was $0.15 \%$ (assuming that no neonates who transferred to VNCH on another indication received a subsequent HIE comorbidity diagnosis). The neonates at Phu San's maternity hospital who require cooling therapy are transferred to VNCH. Only one neonate who received PPV was transferred during the time period when cooling was still indicated (within six hours from birth). We confirmed that this neonate did indeed receive therapeutic hypothermia, which accounts for $0.022 \%$ of the total number of neonates born during the study period. As described above, it is difficult to know the true incidence of HIE. The original definition only included term neonates. However, the understanding has lately moved towards also including preterm neonates, even if a homogenous definition is still lacking [5]. We have decided to report HIE among all neonates. A systematic review, mostly including HIC and a mix of studies including term and/or preterm neonates, estimated the range to be between 0.1 and $0.8 \%$ [32]. One study based in Uganda that included term neonates with a birth weight $>2000 \mathrm{~g}$ reported an HIE incidence of 3.6\% [33]. Another study based in Tanzania that included all neonates reported an HIE incidence of $10.7 \%$ [34].

Despite the difficulty of defining intrapartum-related events, the above data indicate a low rate of these events at Phu San Hanoi Hospital. This finding is difficult to interpret. We will discuss some possible explanations. We have noted a consistently high c-section rate, over $50 \%$ of all births. Previous studies have reported a directly increased mortality (neonatal and maternal) if the c-section rate is below 5\%, and a possible beneficial effect in c-section rates up to 10 to 15\% [35-37]. The WHO stated in 2016 that, due to a lack of data, it was not possible to draw conclusions on the effect on stillbirths and perinatal morbidity when c-section rates exceeded 10\% [35]. A high c-section rate in Vietnam has been reported previously. A study in 2014 looked at 1350 women using a representative sample of households at a national level and found that urban areas had nearly twice the c-section rate (42\%) compared to rural (23\%) [38]. A study from Da Nang, the third largest city in Vietnam, reported a c-section rate of $58 \%$ in public hospitals and $70 \%$ in private hospitals [39]. Another study from Nha Trang, a tourist city in southern Vietnam, reported a c-section rate of $44 \%$ [40]. The high proportion of emergency c-sections at Phu San Hanoi Hospital might indicate a lower-than-average threshold for converting an unsatisfactory vaginal delivery progress to c-section. We report that only one neonate was assisted by instrumental delivery (forceps). Previous studies have reported an instrumental delivery rate between 5 and 20\%, with a failure rate of 5 to $10 \%$ [41]. An indirect measure of good obstetric care is the stillbirth rate. We report a stillbirth rate of $1.0 \%$. The average stillbirth rate in an HIC has previously been reported to be $0.3 \%$, and the stillbirth rate in sub-Saharan Africa and southern Asia has been estimated to approximate 3\% [15]. Moreover, proper antenatal care can contribute to fewer intrapartum-related events. One study reported that the average number of antenatal care visits for women in Vietnam was 7.7 visits in urban areas and 4.4 visits in rural areas [42]. The WHO recently recommended a minimum of eight antenatal visits [43]. We did not report the number of antenatal care visits in our study as this information was not retrospectively available.

We found an $8 \%$ prematurity rate. The WHO reported in 2018 a prematurity rate ranging between $5 \%$ and $18 \%$ [44]. The most common complications among our preterm neonates admitted to the neonatal unit include respiratory distress, feeding difficulties, jaundice, and hospital-acquired infection. These mirror complications that are commonly reported in preterm neonates in both HIC and LIC [13,14,44-46]. Neonates with a birth weight $<1000 \mathrm{~g}$ had a survival rate of $55 \%$, which is more similar to previous reports from northern India (52\%) than reports from Japan (70\%) and Hong Kong (79\%) [47-49]. 
The overall hospital rate of congenital malformations was 1.5\% in inborn neonates. A recent study in Da Nang, an urban hub in central Vietnam, reported a congenital anomaly rate of $3.8 \%$. Even though this rate is somewhat higher than what we report, the distribution was similar, with approximately $50 \%$ of the anomalies affecting the cardiovascular system [50]. The actual incidence at Phu San could be higher if some affected neonates were initially missed, as any malformation is an indication for neonatal unit admission at Phu San. A large study in south India, of 36,074 neonates over 10 years, found an incidence of malformation of $1.3 \%$, with the majority being musculoskeletal (affecting bone and muscle development in the skull, trunk, or limbs, where congenital talipes equinovarus comprised $74 \%$ of the musculoskeletal group). The same study reported congenital anomalies in $9.8 \%$ of the stillbirths [51]. We did not specifically assess stillbirths in relation to congenital anomalies at Phu San.

We report a culture-confirmed nosocomial infection rate of $2.8 \%$ among the neonates admitted to the neonatal unit. When suspected infections (based on risk factors, clinical exam, blood tests, and radiology) are included, the rate increases to $8.1 \%$. Previous studies in the US and Taiwan have reported a nosocomial infection rate around $11 \%$ within the neonatal unit $[52,53]$. As opposed to nosocomial infection rates, the congenital infection rate should not be affected by neonatal transfer. We found culture- or radiology-verified suspected congenital infection in $0.15 \%$ of the neonates born during the study period. This is similar to previous reports from within the USA [17]. The TORCH infection rate was $0.13 \%$ of the births. These infections are known to increase incidences of LBW, congenital anomalies, and stillbirths. Its rate varies among countries and is highly dependent on implemented screening programs [54].

Finally, our observed mortality rate was $0.15 \%$. However, the actual mortality rate depends on the mortality rate of the transferred neonates and the 20 neonates for whom the final discharge location is unknown. Thus, our actual mortality rate lies between 0.15 and $2.2 \%$. We assume, however, that a minority of the transferred and unknown discharge location neonates died, and, therefore, the true figure to be closer to the one we report. Any future study should include neonatal follow-up at VNCH.

\section{Limitations}

Our study has a number of limitations. Its retrospective design carries the limitations of bias always associated with such studies. The cross-sectional period was restricted to one month, which may not mirror the seasonal variation, although the month was carefully selected to be as representative as possible. The sickest neonates at Phu San neonatal unit are transferred to another hospital, which has likely affected our reported infection and mortality rates. This differs from the data presented on intrapartum-related events, prematurity, and congenital anomalies, where it would be difficult to see any transfer-related bias. Finally, as we only looked at the hard copy medical records for the neonates who were admitted to the neonatal unit, there could be a certain degree of underreporting here (in case a neonate was not admitted, for some reason, despite having an admission indication).

\section{Conclusions}

The main local causes of neonatal morbidity and mortality at Phu San Hanoi Hospital were prematurity, infection, congenital malformation, and intrapartum-related events. This is consistent with the leading causes of neonatal adverse outcomes on a global scale. The intrapartum-related events, however, were found to be surprisingly low even in comparison to HIC. A prospective study focusing on intrapartum-related events and immediate resuscitation is necessary for confirming the findings of this study. If a future study can confirm the low rate of intrapartum-related events, attempts should be made to describe the perinatal care at Phu San Hanoi Hospital. Addressing intrapartum-related events and learning how to prevent them will benefit neonates and their families globally. 
Author Contributions: Conceptualization, T.D., P.T.T.P., T.K.N., F.C., D.T., S.M.H., N.P., M.B., L.O., H.V. and T.A.; methodology, T.D., H.L.N., H.T.N., X.A.B., P.T.T.P., T.K.N., S.M.H., N.P., L.O., H.V. and T.A.; validation, F.C., S.M.H., N.P., L.O., H.V. and T.A.; formal analysis, T.D. and F.C.; investigation, H.L.N., H.T.N. and X.A.B.; resources, P.T.T.P., H.V., A.D.N. and T.A.; data curation, T.D., H.L.N., H.T.N. and X.A.B.; writing-original draft preparation, T.D.; writing-review and editing, T.D., H.L.N., H.T.N., X.A.B., P.T.T.P., T.K.N., F.C., D.T., S.M.H., N.P., M.B., L.O., H.V., A.D.N. and T.A.; visualization, T.D.; supervision, P.T.T.P., T.K.N., F.C., D.T., S.M.H., N.P., M.B., L.O., H.V., A.D.N. and T.A.; project administration, T.D., P.T.T.P., T.K.N., S.M.H., N.P., L.O., H.V., A.D.N. and T.A.; funding acquisition, T.K.N., S.M.H., N.P., M.B., L.O., H.V. and T.A. All authors have read and agreed to the published version of the manuscript.

Funding: This study is part of a larger project that has received a Swedish Research Council (Vetenskapsrådet, VR) grant (2018-02770). No funding or benefits were received from any commercial sectors for this study. The Swedish Research Council has no direct role in any technical or intellectual aspect of this work.

Informed Consent Statement: Not applicable.

Data Availability Statement: The data presented in this study are available on request from the corresponding author. The data are not publicly available due to a lack of specification in the ethical application and approval.

Acknowledgments: We thank Aislinn Cook for patiently answering questions concerning RedCap and improving the final RedCap design. We thank Phuong Phung and Phan Nguyen for inserting the hard copy data into RedCap. We thank Tung Truong for supporting all of the technical issues in Vietnam.

Conflicts of Interest: The authors declare that they have no competing interests.

$\begin{array}{ll}\text { Abbreviations } \\ \text { CRF } & \begin{array}{l}\text { case report form } \\ \text { endotracheal tube intubation }\end{array} \\ \text { ETI } & \begin{array}{l}\text { positive pressure ventilation } \\ \text { PPV }\end{array} \\ \text { HIE } & \text { hypoxic ischemic encephalopathy } \\ \text { HIC } & \text { high income countries } \\ \text { LIC } & \text { low income countries } \\ \text { LMIC } & \text { low-middle income countries } \\ \text { UN } & \text { United Nations } \\ \text { WHO } & \text { World Health Organization } \\ \text { VNCH } & \text { Vietnam National Children's Hospital } \\ \text { NBW } & \text { normal birth weight } \\ \text { LBW } & \text { low birth weight } \\ \text { VLBW } & \text { very low birth weight } \\ \text { ELBW } & \text { extremely low birth weight } \\ \text { RDS } & \text { respiratory distress syndrome } \\ \text { PROM } & \text { prolonged ( } \geq 18 \text { h) rupture of membranes }\end{array}$

\section{References}

1. United Nation's Inter-Agency Group for Child Mortality. Levels \& Trends in Child Mortality. 2019. Available online: https: //www.unicef.org/media/60561/ file/UN-IGME-child-mortality-report-2019.pdf (accessed on 4 May 2021).

2. World Health Organization; UNICEF. Ending Preventable Newborn Deaths and Stillbirths by 2030. 2020. Available online: https:/ / www.unicef.org/reports/ending-preventable-newborn-deaths-stillbirths-quality-health-coverage-2020-2025 (accessed on 16 February 2022).

3. Liu, L.; Oza, S.; Hogan, D.; Chu, Y.; Perin, J.; Zhu, J.; Lawn, J.E.; Cousens, S.; Mathers, C.; Black, R.E. Global, regional, and national causes of under-5 mortality in 2000-15: An updated systematic analysis with implications for the Sustainable Development Goals. Lancet 2016, 388, 3027-3035. [CrossRef]

4. Rainaldi, M.A.; Perlman, J.M. Pathophysiology of Birth Asphyxia. Clin. Perinatol. 2016, 43, 409-422. [CrossRef] [PubMed]

5. Gopagondanahalli, K.R.; Li, J.; Fahey, M.; Hunt, R.W.; Jenkin, G.; Miller, S.; Malhotra, A. Preterm Hypoxic-Ischemic Encephalopathy. Front. Pediatr. 2016, 4, 114. [CrossRef] [PubMed] 
6. Azzopardi, D.V.; Strohm, B.; Edwards, A.D.; Dyet, L.; Halliday, H.; Juszczak, E.; Kapellou, O.; Levene, M.; Marlow, N.; Porter, E.; et al. Moderate Hypothermia to Treat Perinatal Asphyxial Encephalopathy. N. Engl. J. Med. 2009, 361, 1349-1358. [CrossRef]

7. Tran, H.T.T.; Le, H.T.T.; Tran, H.T.P.; Khu, D.T.K.; Lagercrantz, H.; Tran, D.M.; Winbladh, B.; Hellström-Westas, L.; Alfvén, T.; Olson, L. Hypothermic treatment for neonatal asphyxia in low-resource settings using phase-changing material-An easy to use and low-cost method. Acta Paediatr. 2021, 110, 85-93. [CrossRef] [PubMed]

8. Bayih, W.A.; Yitbarek, G.Y.; Aynalem, Y.A.; Abate, B.B.; Tesfaw, A.; Ayalew, M.Y.; Belay, D.M.; Hailemeskel, H.S.; Alemu, A.Y. Prevalence and associated factors of birth asphyxia among live births at Debre Tabor General Hospital, North Central Ethiopia. BMC Pregnancy Childbirth 2020, 20, 653. [CrossRef] [PubMed]

9. Peebles, P.J.; Duello, T.M.; Eickhoff, J.C.; McAdams, R.M. Antenatal and intrapartum risk factors for neonatal hypoxic ischemic encephalopathy. J. Perinatol. 2019, 40, 63-69. [CrossRef]

10. Wyckoff, M.H.; Wyllie, J.; Aziz, K.; de Almeida, M.F.; Fabres, J.W.; Fawke, J.; Guinsburg, R.; Hosono, S.; Isayama, T.; Kapadia, V.S.; et al. Neonatal Life Support 2020 International Consensus on Cardiopulmonary Resuscitation and Emergency Cardiovascular Care Science With Treatment Recommendations. Circulation 2020, 142, S185-S221. [CrossRef]

11. Ersdal, H.L.; Eilevstjønn, J.; Linde, J.E.; Yeconia, A.; Mduma, E.R.; Kidanto, H.; Perlman, J. Fresh stillborn and severely asphyxiated neonates share a common hypoxic-ischemic pathway. Int. J. Gynecol. Obstet. 2018, 141, 171-180. [CrossRef]

12. Arya, S.; Naburi, H.; Kawaza, K.; Newton, S.; Anyabolu, C.H.; Bergman, N.; Rao, S.P.N.; Mittal, P.; Assenga, E.; Gadama, L.; et al Immediate "Kangaroo Mother Care" and Survival of Infants with Low Birth Weight. N. Engl. J. Med. 2021, 384, 2028-2038. [CrossRef]

13. Asadi, S.; Bloomfield, F.H.; Harding, J.E. Nutrition in late preterm infants. Semin. Perinatol. 2019, 43, 151160. [CrossRef] [PubMed]

14. Glass, H.C.; Costarino, A.T.; Stayer, S.A.; Brett, C.M.; Cladis, F.; Davis, P.J. Outcomes for Extremely Premature Infants. Anesthesia Analg. 2015, 120, 1337-1351. [CrossRef] [PubMed]

15. Aminu, M.; Bar-Zeev, S.; White, S.; Mathai, M.; Broek, N.V.D. Understanding cause of stillbirth: A prospective observational multi-country study from sub-Saharan Africa. BMC Pregnancy Childbirth 2019, 19, 470. [CrossRef]

16. Goldenberg, R.L.; Harrison, M.S.; McClure, E.M. Stillbirths: The Hidden Birth Asphyxia-US and Global Perspectives. Clin. Perinatol. 2016, 43, 439-453. [CrossRef]

17. Simonsen, K.A.; Anderson-Berry, A.L.; Delair, S.F.; Davies, H.D. Early-onset neonatal sepsis. Clin. Microbiol. Rev. 2014, $27,21-47$. [CrossRef] [PubMed]

18. World Health Organization. Congenital Anomalies. 2020. Available online: https://www.who.int/news-room/fact-sheets/ detail/congenital-anomalies (accessed on 11 May 2021).

19. Ajao, A.E.; Adeoye, I.A. Prevalence, risk factors and outcome of congenital anomalies among neonatal admissions in OGBOMOSO, Nigeria. BMC Pediatr. 2019, 19, 88. [CrossRef] [PubMed]

20. Boyle, B.; Addor, M.-C.; Arriola, L.; Barisic, I.; Bianchi, F.; Csáky-Szunyogh, M.; Walle, H.E.K.D.; Dias, C.M.; Draper, E.; Gatt, M.; et al. Estimating Global Burden of Disease due to congenital anomaly: An analysis of European data. Arch. Dis. Child.-Fetal Neonatal Ed. 2017, 103, F22-F28. [CrossRef]

21. The World Bank. Neonatal Mortality Rate Updated. 2021. Available online: https://data.worldbank.org/indicator/SH.DYN. NMRT?locations=XD-XM (accessed on 17 May 2021).

22. Målqvist, M. Neonatal mortality: An invisible and marginalised trauma. Glob. Health Action 2011, 4, 5724. [CrossRef]

23. Unicef; WHO. Reaching Every Newborn National 2020 Milestones. 2018. Available online: https://www.unicef.org/reports/ committing-child-survival-promise-renewed (accessed on 14 November 2021).

24. Sweet, D.G.; Carnielli, V.; Greisen, G.; Hallman, M.; Ozek, E.; Pas, A.T.; Plavka, R.; Roehr, C.C.; Saugstad, O.D.; Simeoni, U.; et al. European Consensus Guidelines on the Management of Respiratory Distress Syndrome-2019 Update. Neonatology 2019, 115, 432-450. [CrossRef]

25. Lee, A.C.; Cousens, S.; Wall, S.N.; Niermeyer, S.; Darmstadt, G.L.; Carlo, A.W.; Keenan, W.J.; Bhutta, A.Z.; Gill, C.; Lawn, E.J. Neonatal resuscitation and immediate newborn assessment and stimulation for the prevention of neonatal deaths: A systematic review, meta-analysis and Delphi estimation of mortality effect. BMC Public Health 2011, 11, S12. [CrossRef]

26. Niles, D.E.; Cines, C.; Insley, E.; Foglia, E.E.; Elci, O.U.; Skåre, C.; Olasveengen, T.; Ades, A.; Posencheg, M.; Nadkarni, V.M.; et al. Incidence and characteristics of positive pressure ventilation delivered to newborns in a US tertiary academic hospital. Resuscitation 2017, 115, 102-109. [CrossRef] [PubMed]

27. Skåre, C.; Boldingh, A.-M.; Nakstad, B.; Calisch, T.E.; Niles, D.E.; Nadkarni, V.M.; Kramer-Johansen, J.; Olasveengen, T.M. Ventilation fraction during the first $30 \mathrm{~s}$ of neonatal resuscitation. Resuscitation 2016, 107, 25-30. [CrossRef] [PubMed]

28. Skåre, C.; Kramer-Johansen, J.; Steen, T.; Ødegaard, S.; Niles, D.E.; Nakstad, B.; Solevåg, A.L.; Nadkarni, V.M.; Olasveengen, T.M. Incidence of Newborn Stabilization and Resuscitation Measures and Guideline Compliance during the First Minutes of Life in Norway. Neonatology 2015, 108, 100-107. [CrossRef]

29. Souza, J.; Leite, J.M.; Sanudo, A.; Guinsburg, R.; De Sousa, J.R.P. Factors associated with the need for ventilation at birth of neonates weighing $\geq 2500 \mathrm{~g}$. Clinics 2016, 71, 381-386. [CrossRef]

30. Ersdal, H.L.; Mduma, E.; Svensen, E.; Perlman, J. Birth Asphyxia: A Major Cause of Early Neonatal Mortality in a Tanzanian Rural Hospital. Pediatrics 2012, 129, e1238-e1243. [CrossRef]

31. Pejovic, N.J.; Höök, S.M.; Byamugisha, J.; Alfvén, T.; Lubulwa, C.; Cavallin, F.; Nankunda, J.; Ersdal, H.; Blennow, M.; Trevisanuto, D.; et al. A Randomized Trial of Laryngeal Mask Airway in Neonatal Resuscitation. N. Engl. J. Med. 2020, 383, 2138-2147. [CrossRef] 
32. Kurinczuk, J.J.; White-Koning, M.; Badawi, N. Epidemiology of neonatal encephalopathy and hypoxic-ischaemic encephalopathy. Early Hum. Dev. 2010, 86, 329-338. [CrossRef]

33. Namusoke, H.; Nannyonga, M.M.; Ssebunya, R.; Nakibuuka, V.K.; Mworozi, E. Incidence and short term outcomes of neonates with hypoxic ischemic encephalopathy in a Peri Urban teaching hospital, Uganda: A prospective cohort study. Matern. Health Neonatol. Perinatol. 2018, 4, 1-6. [CrossRef]

34. Simiyu, I.N.; Mchaile, D.N.; Katsongeri, K.; Philemon, R.N.; Msuya, S.E. Prevalence, severity and early outcomes of hypoxic ischemic encephalopathy among newborns at a tertiary hospital, in northern Tanzania. BMC Pediatr. 2017, 17, 131. [CrossRef]

35. Betran, A.P.; Torloni, M.R.; Zhang, J.J.; Gulmezoglu, A.M.; Aleem, H.; Althabe, F.; Bergholt, T.; De Bernis, L.; Carroli, G.; Deneux-Tharaux, C.; et al. WHO Statement on Caesarean Section Rates. BJOG Int. J. Obstet. Gynaecol. 2015, 123, 667-670. [CrossRef]

36. Ye, J.; Zhang, J.; Mikolajczyk, R.; Torloni, M.R.; Gülmezoglu, A.M.; Betran, A.P. Association between rates of caesarean section and maternal and neonatal mortality in the 21st century: A worldwide population-based ecological study with longitudinal data. BJOG Int. J. Obstet. Gynaecol. 2015, 123, 745-753. [CrossRef] [PubMed]

37. Cavallaro, F.L.; Cresswell, J.; França, G.V.; Victora, C.; Barros, A.; Ronsmans, C. Trends in caesarean delivery by country and wealth quintile: Cross-sectional surveys in southern Asia and sub-Saharan Africa. Bull. World Health Organ. 2013, 91, 914D-922D. [CrossRef] [PubMed]

38. de Loenzien, M.; Schantz, C.; Luu, B.N.; Dumont, A. Magnitude and correlates of caesarean section in urban and rural areas: A multivariate study in Vietnam. PLoS ONE 2019, 14, e0213129. [CrossRef] [PubMed]

39. Giang, H.T.N.; Ulrich, S.; Tran, H.T.; Pozza, S.B.-D. Monitoring and interventions are needed to reduce the very high Caesarean section rates in Vietnam. Acta Paediatr. 2018, 107, 2109-2114. [CrossRef] [PubMed]

40. Takegata, M.; Ronsmans, C.; Nguyen, H.A.T.; Kitamura, N.; Iwasaki, C.; Toizumi, M.; Moriuchi, H.; Dang, D.A.; Yoshida, L.-M Socio-demographic factors of cesarean births in Nha Trang city, Vietnam: A community-based survey. Trop. Med. Health 2020, 48, 1-8. [CrossRef] [PubMed]

41. Aiken, C.E.; Aiken, A.R.; Brockelsby, J.C.; Scott, J.G. Factors Influencing the Likelihood of Instrumental Delivery Success. Obstet. Gynecol. 2014, 123, 796-803. [CrossRef]

42. Tran, T.K.; Nguyen, C.T.; Nguyen, H.D.; Eriksson, B.; Bondjers, G.; Gottvall, K.; Ascher, H.; Petzold, M. Urban-rural disparities in antenatal care utilization: A study of two cohorts of pregnant women in Vietnam. BMC Health Serv. Res. 2011, 11, 120. [CrossRef]

43. Ekholuenetale, M. Prevalence of Eight or More Antenatal Care Contacts: Findings From Multi-Country Nationally Representative Data. Glob. Pediatr. Health 2021, 8, 2333794X211045822. [CrossRef]

44. World Health Organization. Preterm Birth 2018. Available online: https://www.who.int/news-room/fact-sheets/detail/ preterm-birth (accessed on 19 May 2021).

45. Darmaun, D.; Lapillonne, A.; Simeoni, U.; Picaud, J.-C.; Rozé, J.-C.; Saliba, E.; Bocquet, A.; Chouraqui, J.-P.; Dupont, C.; Feillet, F.; et al. Parenteral nutrition for preterm infants: Issues and strategy. Arch. Pédiatrie 2018, 25, 286-294. [CrossRef]

46. Manuck, T.A.; Rice, M.M.; Bailit, J.L.; Grobman, W.A.; Reddy, U.M.; Wapner, R.; Thorp, J.M.; Caritis, S.N.; Prasad, M.; Tita, A.; et al. Preterm neonatal morbidity and mortality by gestational age: A contemporary cohort. Am. J. Obstet. Gynecol. 2016, 215, 103. [CrossRef]

47. Hon, K.; Liu, S.; Chow, J.C.; Tsang, K.Y.; Lam, H.; So, K.; Cheng, Y.K.; Leung, A.K.; Wong, W. Mortality and morbidity of extremely low birth weight infants in Hong Kong, 2010-2017: A single-centre review. Hong Kong Med. J. 2018, $24,460-465$. [CrossRef] [PubMed]

48. Itabashi, K.; Horiuchi, T.; Kusuda, S.; Kabe, K.; Itani, Y.; Nakamura, T.; Fujimura, M.; Matsuo, M. Mortality Rates for Extremely Low Birth Weight Infants Born in Japan in 2005. Pediatrics 2009, 123, 445-450. [CrossRef] [PubMed]

49. Mukhopadhyay, K.; Louis, D.; Mahajan, R.; Kumar, P. Predictors of mortality and major morbidities in extremely low birth weight neonates. Indian Pediatr. 2013, 50, 1119-1123. [CrossRef] [PubMed]

50. Giang, H.T.N.; Pozza, S.B.-D.; Ulrich, S.; Linh, L.K.; Tran, H.T. Prevalence and Pattern of Congenital Anomalies in a Tertiary Hospital in Central Vietnam. J. Trop. Pediatr. 2019, 66, 187-193. [CrossRef]

51. Cherian, A.G.; Jamkhandi, D.; George, K.; Bose, A.; Prasad, J.; Minz, S. Prevalence of Congenital Anomalies in a Secondary Care Hospital in South India: A Cross-Sectional Study. J. Trop. Pediatr. 2016, 62, 361-367. [CrossRef]

52. Sohn, A.H.; Garrett, D.O.; Sinkowitz-Cochran, R.L.; Grohskopf, L.A.; Levine, G.L.; Stover, B.H.; Siegel, J.D.; Jarvis, W.R. Prevalence of nosocomial infections in neonatal intensive care unit patients: Results from the first national point-prevalence survey. J. Pediatr. 2001, 139, 821-827. [CrossRef]

53. Chen, Y.-C.; Lin, C.-F.; Rehn, Y.-J.F.; Chen, J.-C.; Chen, P.-Y.; Chen, C.-H.; Wang, T.-M.; Huang, F.-L. Reduced nosocomial infection rate in a neonatal intensive care unit during a 4-year surveillance period. J. Chin. Med. Assoc. 2017, 80, 427-431. [CrossRef]

54. Madrid, L.; Varo, R.; Sitoe, A.; Bassat, Q. Congenital and perinatally-acquired infections in resource-constrained settings. Expert Rev. Anti-Infect. Ther. 2016, 14, 845-861. [CrossRef] 